\title{
Valutazione dei bisogni dei pazienti in carico ai servizi territoriali
}

\author{
TOMMASO LOSAVIO
}

I Servizi Territoriali per la Salute Mentale che hanno raccolto, trasformandola radicalmente, l'assistenza dapprima erogata prevalentemente - se non esclusivamente - dal manicomio - nascono in Italia (forse sarebbe meglio dire che sarebbero dovuti nascere) come l'innovazione profonda prodotta proprio da quell'azione che aveva permesso di mettere profondamente in crisi il modello manicomiale. Quell'azione che, è bene ricordarlo, nacque e si sviluppò alcuni anni prima della legge 180 che da essa risultò significativamente determinata, non fu mai né semplice negazione burocratico-amministrativa dell'internamento (la deospedalizzazione intesa soprattutto come dimissioni ad ogni costo per ridurre i costi altissimi dei ricoveri interminabili) né fu mai negazione ideologica della malattia mentale (l'antipsichiatria come espressione radicale, soprattutto della cultura anglosassone, che chiedeva l'abolizione di tutte le istituzioni che esercitavano un controllo sociale).

L'esperienza italiana della psichiatria di comunità, che per molti tratti è significativamente diversa dalle esperienze analoghe ormai universalmente diffuse (in particolare per la cancellazione del manicomio), trova le sue radici in particolare in quel processo, complesso e articolato, che è stato chiamato «deistituzionalizzazione» (Rotelli et. al., 1992).

La scomposizione e la decostruzione «dall'interno» della stessa istituzione psichiatrica ha determinato, e per molti aspetti continua a determinare, la contemporanea costruzione del nuovo sistema della salute mentale come interamente sostitutivo del manicomio, delle sue prassi, ma anche dei riferimenti culturali sui quali manicomio e prassi erano fondati.

È ovvio, quindi, che per istituzione non si può intendere soltanto e semplicemente il luogo fisico rap-

Indirizzo per la corrispondenza: Professor T. Losavio, Servizio Dipartimentale di Salute Mentale, USL RM 12, via P. Gasparri 21, 00168 Roma. presentato dal manicomio ma anche, ed in particolare, l'insieme dei modelli, dei meccanismi, delle operatività, delle culture che, dentro e fuori del manicomio hanno governato, ed in modi più o meno espliciti continuano a governare, nel campo del «Sistema Psichiatria» (Pirella, 1993), i rapporti tra curante e curato, tra problema e soluzione, tra salute e malattia, tra curabilità e incurabilità, tra guarigione e cronicità, tra domande (intese come bisogni) e risposte (intese come pratiche).

La «messa tra parentesi» della malattia mentale - non come posizione ideologica di negazione, ma come azione pratica di non attribuzione di tutto il comportamento del paziente alla malattia -; l'attivazione di risorse, continuamente rinnovate, a favore del paziente e non più accumulate nell'istituzione; l'attribuzione di senso e di valore alla parola del paziente; la radicale trasformazione della dimensione temporale uscita dall'infinitezza istituzionale; il lento e difficile ma tenace attraversamento della comunità, sono alcune delle operazioni fondamentali che hanno fatto da guida al lavoro di deistituzionalizzazione all'interno del manicomio prima, e all'esterno, nell'intervento territoriale, ora.

Il processo trasformativo che in tal modo ha investito i percorsi attraverso cui si articolano le risposte dei servizi ha determinato un processo altrettanto radicalmente innovativo, il cui destino oggi non è facile indovinare, anche della domanda (bisogni) nei suoi meccanismi costitutivi, nelle sue modalità di formulazione, nella concretezza delle sue richieste. Le contraddizioni emergenti dalla concatenazione domandarisposta-domanda-... rendono gli operatori dei Servizi Territoriali partecipi di una mutazione che, a partire dal cambiamento della gestione dei problemi psichiatrici, investe la psichiatria stessa in tutti i suoi aspetti costitutivi dalle sue basi psicopatologiche, alle espressioni cliniche, dalle ipotesi preventive alle evoluzioni prognostiche. 
In questa prospettiva i bisogni dei pazienti si evidenziano come valenze assolutamente nuove e non perché, ovviamente nuovi sono i bisogni, ma perché nuove sono le chiavi di lettura di essi, nuove sono le prospettive di risposta, nuove - e continuamente rinnovabili - possono essere le risorse a disposizione, da attivare, da pretendere.

Se la psichiatria, come disciplina medica tradizionalmente intesa, ha come obiettivo la sanzione dell'incomprensibilità, la categorializzazione della diversità, l'oggettivizzazione della sofferenza ridotta a malattia, la salute mentale (i servizi) è la valorizzazione di ogni singola esperienza in quanto condizione di vita (e non solo di malattia) irripetibile, è la conoscenza dell'uomo e della donna che soffrono nel loro contesto di vita quotidiana con le loro aspettative, le loro frustrazioni, i loro desideri, le loro difficoltà.

La "presa in carico» (Pastore, 1992), pratica su cui dovrebbe fondarsi l'attività dei Servizi, ha fatto sì che il campo d'intervento divenisse la vita stessa della persona sofferente per la costruzione di occasioni di vita e non semplicemente per il silenziamento sintomatologico e per l'adattamento a condizioni di vita considerate immodificabili (cronicità).

La diagnosi stessa acquista valenze del tutto nuove in quest'ambito dell'operare clinico: il suo oggetto non è più né la malattia né la sofferenza, ma la persona malata che soffre. I sintomi psicopatologici diventano indicatori deboli e la conoscenza della soggettività dell'altro acquista i caratteri di un processo aperto che rifugge dalla furia definitoria e chiede pazienza (partecipazione e tempo) all'osservante. Il processo diagnostico si connota inevitabilmente di valenze etiche che danno voce all'osservato, alla sua storia personale, all'interazione tra queste ed il suo abituale contesto di vita. Ma anche «la comunità», nella quale «quella» persona esprime la sua sofferenza e nella quale il servizio agisce la sua pratica, diviene un polo obbligato di un processo di conoscenza: le sue contraddizioni, la possibilità di rispondere ai bisogni delle persone (sane e malate), la capacità di accoglienza e di solidarietà, le occasioni di vita costituiscono parametri di valutazione dai quali non è possibile prescindere in una prospettiva corretta di valutazione dei bisogni del paziente. Essa assume non soltanto valore di atto prevalentemente «etico» che si sovrappone ad un sistema valoriale «tecnico» (Saraceno, 1992), ma si costituisce come crinale tra vecchia e nuova psichiatria, tra oggettivazione e valoriz- zazione della soggettività, tra definizione e proposta.

Da una epidemiologia dei censimenti (De Luca, et al., 1992) attenta a sintomi, diagnosi, farmaci, incidenza e prevalenza è necessario lavorare per un'epidemiologia capace di valutare elementi quali il funzionamento sociale, l'integrazione sociale, l'adattamento personale, la soddisfazione (Lehaman, 1983).

La rilevazione di parametri con una prevalente componente di soggettività pone certamente indubbie difficoltà di misurazione, ma non v'è dubbio che proprio nella soggettività di queste valutazioni risiedono gli aspetti più immotivati della loro rilevanza (Ruggeri, 1992).

La ricerca nel campo della valutazione della qualità della vita (Tantam, 1988; Oliver, 1990; Boatta et al., 1992) del livello di soddisfazione dei pazienti e dei loro familiari, delle aspettative dei pazienti, dei familiari e degli operatori, ha, ritengo, una grande potenzialità di promuovere nuove conoscenze e nuove operatività che si collocano di diritto in quel processo di destituzionalizzazione che, iniziato 30 anni or sono nei manicomi, mostra oggi nei nuovi Servizi Territoriali ancora la capacità di rendere provvisori i nostri saperi.

\section{BIBLIOGRAFIA}

Boatta V., Di Cesare A., Losavio T. \& Quaranta G. (1992). Qualità della vita e assistenza territoriale. Proposte per la Salute Mentale 2, 91-105.

De Luca L., Frattura L. \& Marino S. (1992). Proposte di epidemiologia del silenzio. Inventario di Psichiatria 7, 7-12.

Lehaman A. F. (1983). The well-being of chronic mental patients: assessing their quality o life. Archives of General Psychiatry 40, 369-373.

Olivier J. P. (1990). The quality of life in community care. In Residential Needs for Severaly Disabled Psychiatric Patients (ed. K. Wooff). Salford: London.

Pastore V. (1992). Servizi/solidarietà/supporto sociale. Epidemiologia e Psichiatria Sociale 1, 157-159.

Pirella A. (1993). Il sistema psichiatria. Convegno di Psichiatria Democratica. Livorno (in stampa).

Rotelli F., De Leonardis O. \& Marzi D. (1992). Destituzionalizzazione, un'altra via. Fogli d'Informazione 157, 54-77.

Ruggeri M. (1992). Aspettative e soddisfazione dei pazienti e dei loro familiari verso i servizi psichiatrici. Proposte per la Salute Mentale 2, 107-129.

Saraceno B. (1992). Vere e false differenze. Fogli di Informazione $157,117-124$.

Tantam D. (1988). Quality of life and the chronically mental ill. International Journal of Social Psychiatry 34, 243-247. 